\title{
PREDOMINANCE AND INFLUENCE OF VITAMIN D DEFICIENCY ON GLYCEMIC AND LIPID INDICES IN TYPE 2 DIABETES PATIENTS: A CASE-CONTROL STUDY
}

\author{
DURGARAO Y ${ }^{1}$, POORNIMA A MANJREKAR ${ }^{1 *}$, PRABHA ADHIKARI ${ }^{2}$, ARUN ${ }^{3}$, CHAKRAPANI ${ }^{3}{ }^{3}$, RUKMINI MS ${ }^{1}$
}

${ }^{1}$ Department of Biochemistry, Kasturba Medical College, Mangaluru, Karnataka, India. ${ }^{2}$ Department of Internal Medicine, Yenepoya University, Mangalore, Karnataka, India. 3Department of Internal Medicine, Kasturba Medical College, Mangaluru, Manipal University, Karnataka, India. Email: drpamanjrekar@gmail.com

Received: 01 December 2016, Revised and Accepted: 16 January 2017

ABSTRACT

Objective: To investigate the existence of vitamin D deficiency (VDD) among Type 2 diabetes, nondiabetes individuals and its effect on both glycemic and lipid profiles.

Methods: A case-control study was conducted on 200 subjects of both genders (100 Type 2 diabetes and 100 nondiabetes individuals) aged 40-60 years. Fasting serum 25-hydroxycholecalciferol (25(OH) D) levels, fasting blood sugar (FBS), hemoglobin A1c (HbA1c), lipid profile including total cholesterol (TC), triglycerides (TGs), high density lipoprotein (HDL), low density lipoprotein (LDL), TC/HDL, and very LDL were estimated. Atherogenic index of plasma (AIP) was calculated. Group comparisons were done by one-way analysis of variance followed by post-hoc Tukey's test and Student's independent t-test. Chi-square test was performed for categorical variables. Correlation was done by Pearson's analysis. p<0.05 was considered significant.

Results: The average serum 25(OH) D levels were significantly $(\mathrm{p}<0.001)$ low in diabetes group. The prevalence of VDD and the percentage of insufficient and sufficient categories was significantly $(\mathrm{p}<0.001)$ high and low, respectively, in diabetes group. In the deficient category, diabetes group had severe VDD with significantly low-HDL and elevated TGs and there was an insignificant but negative association between serum vitamin D levels, FBS, HbA1c, TC, TG, LDL, TC/HDL, and AIP among diabetes patients.

Conclusion: The occurrence of severe VDD coupled with the independent association of the same with the glycemic and lipid profiles in Type 2 diabetes may further add to the aggravation of complications

Keywords: Vitamin D deficiency, Type 2 diabetes, Glycemic and lipid indices.

(c) 2017 The Authors. Published by Innovare Academic Sciences Pvt Ltd. This is an open access article under the CC BY license (http://creativecommons. org/licenses/by/4. 0/) DOI: http://dx.doi.org/10.22159/ajpcr.2017.v10i4.16437

\section{INTRODUCTION}

Vitamin D deficiency (VDD) is a globally widespread pandemic [1] that affects the functions of various physiological systems. Over the past decade, the extraskeletal effects of vitamin D are gaining importance. The discovery of vitamin $\mathrm{D}$ receptors (VDRs) in various organs coupled with the local synthesis of vitamin D strengthen this concept further [2]. VDD could well be a new risk factor for many diseases such as hypertension [3], cardiovascular disease [4,5], Type 1 and Type 2 diabetes [6], immune disorders, osteoporosis, and cancer [7].

Over 50\% Type 2 diabetes mellitus (T2DM) patients have $<20 \mathrm{ng} / \mathrm{L}$ of serum 25 hydroxy vitamin D (25(OH) D) level [8]. The American Diabetic Association and the American Heart Association strongly recommended the control of nonglycemic risk factors in diabetes patients to halt the onset as well as propensity of various complications [1]. Studies regarding the influence of serum vitamin $\mathrm{D}$ levels on diabetes and its associated dyslipidemia yielded conflicting results [9-11] and there are few comparative studies on the prevalence of VDD among individuals with and without diabetes [12]. In an attempt to address the same, this study is aimed to investigate the existence of VDD among diabetes patients as compared to nondiabetes individuals and its effect on both glycemic status and lipid profile.

\section{METHODS}

This is a hospital-based case-control study conducted on 200 subjects of both genders (100 diabetes and 100 nondiabetes individuals) aged 40-60 years. The study protocol was approved by Institutional Ethics Committee (No. IEC KMC MLR 02-14/27) and the participants were enrolled after obtaining duly signed informed consent. Individuals who were on vitamin D supplementation and subjects with any acute or chronic illness as documented by history were excluded from the study. Fasting serum 25(OH) D levels (Diasource, USA) by enzyme-linked immunosorbent assay, fasting blood sugar (FBS), lipid profile including total cholesterol (TC), triglycerides (TG), high density lipoprotein (HDL), low density lipoprotein (LDL), TC/HDL, and very LDL (VLDL) were estimated using COBAS - 600 autoanalyzer. Hemoglobin A1c (HbA1c) estimation was done in D-10 BioRad analyzer. Atherogenic index of plasma (AIP) was calculated with Dobiasova M. calculator [Log (TG/HDL-C)]. Based on the serum 25(OH) D levels the study population was divided into three groups as per Institute of Medicine guidelines. Deficient $<20 \mathrm{ng} / \mathrm{mL}$, insufficient 21-29 ng/mL, and sufficient $>30 \mathrm{ng} / \mathrm{mL}$.

\section{Statistical analysis}

The data were analyzed in SPSS V 17.0. The results were expressed as mean \pm standard deviation, median and interquartiles. Comparison between the groups was done by analysis of variance followed by Tukey's multiple comparison test and Student's independent t-test. Categorical variables were analyzed by Chi-square test. The correlation was done by Pearson's analysis. $\mathrm{p}<0.05$ was considered significant.

\section{RESULTS}

Among diabetes and nondiabetic groups the mean age and male:female ratio was found to be $49.76 \pm 5.21$ years versus $48.52 \pm 6.03$ years and 63:37 versus $66: 34$, respectively. The average serum $25(\mathrm{OH}) \mathrm{D}$ levels were significantly $(\mathrm{p}<0.001)$ low in diabetes group $(9[5,14]$ vs. $19[12,28]) \mathrm{ng} / \mathrm{mL}$ (Tables 1 and 2). Compared to nondiabetes group 
Table 1: Parameters of diabetes subjects compared among vitamin D categories

\begin{tabular}{llll}
\hline Parameters & $\mathbf{2 0}(\mathbf{n = 8 6})$ & $\mathbf{2 0 . 1 - 2 9 . 9}(\mathbf{n = 5})$ & Total $(\mathbf{n = 1 0 0})$ \\
\hline Age & $49.37 \pm 5.24$ & $52.6 \pm 6.06$ & $4 \mathbf{n = 9})$ \\
Vitamin D & $8.51 \pm 4.70$ & $22.82 \pm 1.48$ & $4.88 \pm 3.72$ \\
FBS & $162.18 \pm 51.57$ & $143.4 \pm 28.54$ & $43.93 \pm 10.23$ \\
HbA1c & $8.36 \pm 1.86$ & $7.84 \pm 0.70$ & $160.11 \pm 58.25$ \\
TC & $186.10 \pm 43.81$ & $187.4 \pm 64.98$ & $8.05 \pm 1.48$ \\
TG & $164.16 \pm 86.10$ & $108.8 \pm 32.32$ & $185.55 \pm 31.12$ \\
HDL & $40.81 \pm 9.27$ & $45.86 \pm 11.65$ & $149.55 \pm 64.09$ \\
LDL & $129.76 \pm 38.72$ & $133.98 \pm 66.21$ & $47.91 \pm 18.20$ \\
TC/HDL & $4.72 \pm 1.39$ & $4.24 \pm 1.52$ & $124.22 \pm 29.30$ \\
AIP & $0.23 \pm 0.19$ & $0.05 \pm 0.06$ & $4.26 \pm 1.38$ \\
VLDL & $32.83 \pm 17.2$ & $21.74 \pm 6.47$ & $0.18 \pm 0.21$ \\
Male:Female & $53: 33$ & $3: 2$ & $29.91 \pm 12.81$ \\
\hline
\end{tabular}

Expressed as Mean \pm SD. Groups were compared by one way Anova followed by Tukey's multiple comparison test. Categorical variables were analyzed by chi-square test. FBS: Fasting blood sugar, TC: Total cholesterol, TG: Triglycerides, HDL: High density lipoprotein, LDL: Low density lipoprotein, AIP: Atherogenic index of plasma, VLDL: Very low density lipoprotein, HbA1c: Hemoglobin A1c

Table 2: Study parameters in non-diabetes group compared among vitamin D categories

\begin{tabular}{|c|c|c|c|c|}
\hline Parameters & $<20(n=54)$ & $21-29(n=25)$ & $>30(n=21)$ & Total $(n=100)$ \\
\hline Age & $48.29 \pm 6.35$ & $48.88 \pm 5.90$ & $48.66 \pm 5.59$ & $48.52 \pm 6.03$ \\
\hline Vitamin D & $11.77 \pm 4.92$ & $24.26 \pm 3.05$ & $45.57 \pm 13.78$ & $19(12,28)^{*}$ \\
\hline FBS & $96.77 \pm 8.12$ & $98.4 \pm 9.03$ & $98.28 \pm 9.07$ & $97.5 \pm 8.51$ \\
\hline $\mathrm{HbA1c}$ & $5.48 \pm 0.42$ & $5.62 \pm 0.53$ & $5.67 \pm 0.37$ & $5.56 \pm 0.44$ \\
\hline TC & $197.53 \pm 37.17$ & $194 \pm 31.60$ & $197.23 \pm 31.74$ & $196.59 \pm 34.47$ \\
\hline TG & $128.03 \pm 60.21$ & $120.84 \pm 54.23$ & $120.33 \pm 37.43$ & $124.62 \pm 54.32$ \\
\hline HDL & $49.04 \pm 12.12$ & $52.01 \pm 13.68$ & $48.08 \pm 13.62$ & $49.58 \pm 12.79$ \\
\hline TC/HDL & $4.19 \pm 0.98$ & $3.93 \pm 1.11$ & $4.45 \pm 1.59$ & $4.18 \pm 1.16$ \\
\hline AIP & $0.12 \pm 0.16$ & $0.09 \pm 0.15$ & $0.10 \pm 0.15$ & $0.11 \pm 0.16$ \\
\hline VLDL & $25.01 \pm 12.33$ & $23.88 \pm 10.84$ & $24.06 \pm 7.48$ & $24.53 \pm 11.02$ \\
\hline Male:Female & $37: 17$ & $13: 12$ & $16: 5$ & $66: 34$ \\
\hline
\end{tabular}

*Expressed as Mean \pm SD. Groups were compared by one way Anova followed by Tukey's multiple comparison test. Categorical variables were analyzed by chi-square test.. FBS: Fasting blood sugar, TC: Total cholesterol, TG: Triglycerides, HDL: High density lipoprotein, LDL: Low density lipoprotein, AIP: Atherogenic index of plasma, VLDL: Very low density lipoprotein, HbA1c: Hemoglobin A1c

the prevalence of VDD was significantly high in diabetes group (54\% vs. $86 \%$ ) and the percentage of insufficient and sufficient categories were also significantly low ( $5 \%$ and $9 \%$ vs. $25 \%$, and $21 \%$ ) respectively in diabetes group (Fig. 1). In the VDD category ( $n=140)$, the ratio of diabetes versus nondiabetic individuals was found to be $86: 54$ and the diabetes group had severe VDD with significantly low-HDL, elevated TGs, TC/HDL ratio, and AIP (Table 3). Although insignificant there was a negative association between serum vitamin D levels FBS, HbA1c, TC, TG, LDL, TC/HDL, and AIP among diabetes patients (Table 4).

\section{DISCUSSION}

VDD is considered as a global pandemic and has been reported in healthy population. More than $90 \%$ of relatively healthy Indians have low $25(\mathrm{OH})$ D levels. The common causes for VDD in apparently healthy individuals are low dietary intake and indoor life style with minimal exposure to sunlight [13]. It has been proposed that low serum vitamin D levels predispose individuals to T2DM [14]. Studies also demonstrated higher prevalence of VDD among diabetes patients compared to nondiabetic individuals [14]. In this study, more than half $(54 \%)$ of the nondiabetic individuals were found to have VDD whereas the extent of VDD was much higher (86\%) in diabetic group (Fig. 1). These findings reiterate the previous reports of widespread VDD even among apparently healthy subjects and those affected by diabetes are more severely affected. Diabetes patients are more prone to VDD has been supported by previous studies where Boucher B et al., Isaia G et al., Mattila C et al., reported correlation of low serum 25(OH) D concentrations with impaired glucose tolerance and an increased risk of Type 2 diabetes [14-16]. Evidence from cross-sectional studies showed that low serum 25-(OH) D levels are linked to impaired glucose tolerance and diabetes [18]. A meta-analysis of 21 prospective studies concluded that lower serum vitamin D levels are associated with hyperglycemia and insulin resistance [19]. The presence of VDR and vitamin D binding proteins (VDBP) in pancreatic tissues

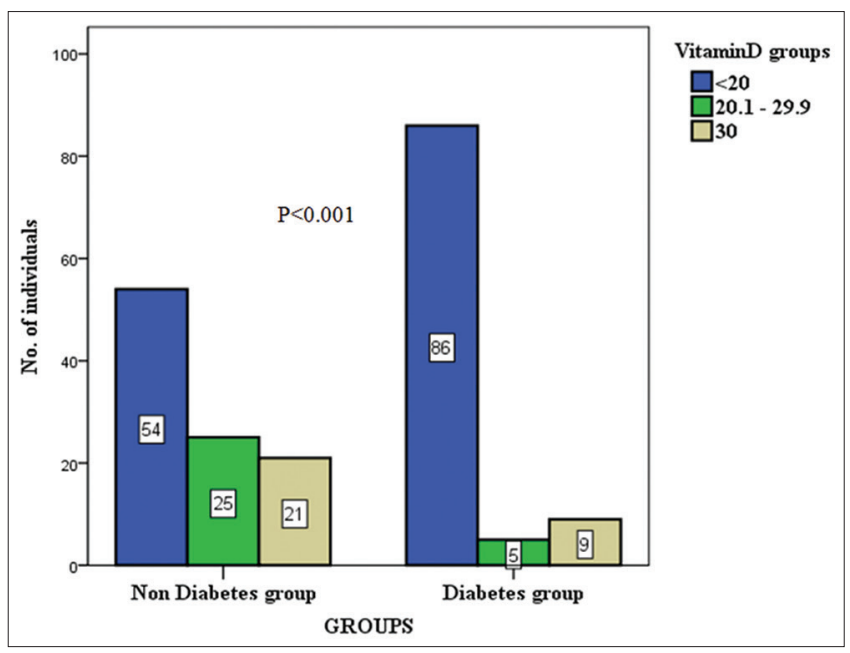

Fig. 1: Comparison of vitamin D status between diabetes and nondiabetes groups

strengthen the concept of vitamin D being essential for insulin synthesis and secretion [14]. The probable mechanisms for VDD among diabetes patients could be obesity, (vitamin D being fat soluble gets sequestered in adipose tissue leading to low serum levels) decreased VDBP secondary to reduced function or availability of megalin or low density lipoproteinrelated protein 2 (hasten the metabolism and elimination of active form of vitamin D) [20].

Vitamin D was proposed to exert favorable actions in Type 2 DM patients through the following pathways, (i) Improved $\beta$-cell function 
Table 3: Comparison of study variables in diabetes and nondiabetes groups of vitamin $\mathrm{D}$ deficient category

\begin{tabular}{llll}
\hline Parameters & DM (n=86) & NDM $(\mathbf{n = 5 4 )}$ & p value \\
\hline Age & $49.37 \pm 5.24$ & $48.29 \pm 6.35$ & 0.30 \\
Male:female & $53: 33$ & $17: 37$ & - \\
Vitamin D & $8.51 \pm 4.70$ & $11.77 \pm 4.92$ & 0.001 \\
FBS & $162.18 \pm 51.57$ & $96.77 \pm 8.12$ & 0.001 \\
HbA1c & $8.36 \pm 1.86$ & $5.48 \pm 0.42$ & 0.001 \\
TC & $186.10 \pm 43.81$ & $197.53 \pm 37.17$ & 0.114 \\
TG & $164.16 \pm 86.10$ & $128.03 \pm 60.21$ & 0.008 \\
HDL & $40.81 \pm 9.27$ & $49.04 \pm 12.12$ & 0.001 \\
LDL & $129.76 \pm 38.72$ & $133.92 \pm 33.87$ & 0.51 \\
TC/HDL & $4.72 \pm 1.39$ & $4.193 \pm 0.98$ & 0.01 \\
AIP & $0.23 \pm 0.199$ & $0.12 \pm 0.16$ & 0.001 \\
VLDL & $32.83 \pm 17.22$ & $25.01 \pm 12.33$ & 0.004 \\
\hline
\end{tabular}

*p-value was obtained through student's independent $\mathrm{t}$ test. FBS: Fasting blood sugar, TC: Total cholesterol, TG: Triglycerides, HDL: High density lipoprotein, LDL: Low density lipoprotein, AIP: Atherogenic index of plasma, VLDL: Very low density lipoprotein, DM: Diabetes mellitus, NDM: Nondiabetes mellitus, HbA1c: Hemoglobin A1c

Table 4: Correlation of vitamin D with glycemic and lipid indices in diabetes subjects

\begin{tabular}{lll}
\hline Variables & r value & p value \\
\hline FBS & -0.01 & 0.88 \\
HbA1c & -0.05 & 0.60 \\
TC & -0.04 & 0.64 \\
TG & -0.10 & 0.30 \\
HDL & 0.12 & 0.20 \\
LDL & -0.05 & 0.60 \\
TC/HDL & -0.09 & 0.37 \\
AIP & -0.09 & 0.33 \\
VLDL & -0.10 & 0.30 \\
\hline
\end{tabular}

The r-value and p-value were calculated by Pearson's correlation analysis Pearson's correlation. FBS: Fasting blood sugar, TC: Total cholesterol,

TG: Triglycerides, HDL: High density lipoprotein, LDL: Low density lipoprotein, AIP: Atherogenic index of plasma, VLDL: Very low density lipoprotein, HbA1c: Hemoglobin A1c

via direct effect of vitamin $\mathrm{D}$ or by increase in the intracellular $\mathrm{Ca}^{2+}$ concentration which in turn boost insulin release, (ii) augmentation of insulin sensitivity in target cells through glucose transporter type 4 translocation and insulin receptor expression via calciumdependent pathways ultimately leading to better glucose utilization and (iii) inhibition of $\beta$-cells apoptosis via VDR transcription factor mediated inhibition of cytotoxic gene expression [21]. In diabetes patients of this study, there was a negative but nonsignificant correlation of glycemic profile with vitamin D status (Table 4). When compared across the vitamin D categories the deficient group had high fasting sugar and HbA1c levels than insufficient and sufficient groups but the difference was not statistically significant. Paradoxically, the insufficient category has better glycemic profile than sufficient group (Table 1). Diabetes group had a significant severe form of VDD compared to the apparently healthy individuals suggesting that this population are at high risk of developing VDD and this is in acceptance with the previous studies [21,22]. In a study conducted on 83,779 women for 20 years reported the role of vitamin D in attenuating the risk of T2DM [23]. It has been shown that restoration of serum vitamin $D$ levels to normal lead to the improvement of glucose tolerance [24,25].

Data from previous studies pointed the role of serum $25(\mathrm{OH}) \mathrm{D}$ levels in dyslipidemia. Nevertheless, the exact mechanism(s) relating VDD with dyslipidemia are not well known and there exist disparity [27]. In the current study, there was no significant difference in serum lipid profile across the vitamin D categories of both the groups (Tables 1 and 2) but in the diabetes group the deficient category had elevated TGs, AIP, and low-HDL compared to other two categories. Similar to the glycemic profile the lipid profile was also better in insufficient category which may be related to the specific pharmacotherapy of these patients. Jorde R, Grimnes G [28] reported a positive correlation between vitamin D status and HDL whereas, Xinyan Bi et al. [11] found no such association but they reported a significant inverse relation between TC/HDL, LDL/HDL ratios and serum 25(OH) D levels. In this study, insignificant but negative and positive associations were reported between serum 25(OH) D levels, TC, TGs, TC/HDL ratio, AIP and HDL, respectively, among the diabetes patients (Table 4). An AIP above 0.5 has been a suggestive of atherogenic risk [29]. Although diabetes patients of deficient category had higher AIP compared to nondiabetes group irrespective of the vitamin D status it is within the specified cut off. Saedisomeolia et al. [30] established a statistically insignificant positive relation between serum $25(\mathrm{OH})$ D levels, LDL, and TC. They also showed an inverse relationship between serum 25(OH) D and TG levels in diabetes individuals. Venkatesh $\mathrm{G}$ et al. showed that exposure to sun light had no significant changes on lipid profile of prediabetes subjects. The authors highlighted the need for appropriate vitamin D supplementation with intensive life style changes for the prevention or delay of T2DM progression [31].

The modulatory effects of vitamin D on lipid profile has been through direct and indirect effects and its role in attenuation of serum TGs may be due to the regulatory action that increases the lipoprotein lipase activity in adiposity [31]. The role of calcium-mediated regulation of cholesterol and other components of lipid profile is not well known. In a study conducted by Querfeld et al. [32] they reported that vitamin D supplementation had a significant effect over specific components of lipid profile and revealed $8 \%(0.28 \mathrm{mmol} / \mathrm{L})$ increase in serum LDL-C and a $16 \%(0.22 \mathrm{mmol} / \mathrm{L})$ decrease in serum TG compared to the placebo group.

The strengths of the study are comparison of serum vitamin D levels between diabetes and nondiabetes individuals with reference to the severity of VDD among diabetes patients with an emphasis on deficient category between the groups. The limitation is the influence of diabetes, drug therapy and other comorbidities on the study variables was not considered.

\section{CONCLUSION}

The occurrence of severe VDD coupled with the negative association of the same although insignificant with the glycemic and lipid profiles excluding HDL in diabetes population may further add to the aggravation of complications in already compromised situation. Hence, maintenance of adequate serum vitamin D levels should be a priority especially in diabetes individuals.

\section{REFERENCES}

1. Chaudhuri JR, Mridula KR, Anamika A, Boddu DB, Misra PK, Lingaiah A, et al. Deficiency of 25-hydroxyvitamin D and dyslipidemia in Indian subjects. J Lipids 2013;2013:623420.

2. Alvarez JA, Ashraf A. Role of vitamin D in insulin secretion and insulin sensitivity for glucose homeostasis. Int J Endocrinol 2010;2010:351-85.

3. Scragg R, Sowers M, Bell C. Serum 25-hydroxyvitamin D, ethnicity, and blood pressure in the third national health and nutrition examination survey. Am J Hypertens 2007;20(7):713-9.

4. Michos ED, Melamed ML. Vitamin D and cardiovascular disease risk. Curr Opin Clin Nutr Metab Care 2008;11(1):7-12.

5. Wang TJ, Pencina MJ, Booth SL, Jacques PF, Ingelsson E, Lanier K, et al. Vitamin D deficiency and risk of cardiovascular disease. Circulation 2008;117(4):503-11.

6. Holick MF. High prevalence of vitamin D inadequacy and implications for health. Mayo Clin Proc 2006;81(3):353-73.

7. Witham MD, Dove FJ, Dryburgh M, Sugden JA, Morris AD, Struthers AD. The effect of different doses of vitamin D(3) on markers of vascular health in patients with Type 2 diabetes: A randomised controlled trial. Diabetologia 2010;53(1):2112-9.

8. Skyler JS, Bergenstal R, Bonow RO, Buse J, Deedwania P, Gale EA, et al. Intensive glycemic control and the prevention of cardiovascular events: Implications of the accord, advance, and VA diabetes trials: A position statement of the American diabetes association and a 
scientific statement of the American college of cardiology foundation and the American heart association. Circulation 2009;19:351-7.

9. Saedisomeolia A, Taheri E, Djalali M, Moghadam AM, Qorbani M. Association between serum level of vitamin D and lipid profiles in Type 2 diabetic patients in Iran. J Diabetes Metab Disord

10. Bouł̊t3Algůrre $P$, Flores $M$, Macias N, Quezada AD, DenovaGutiérrez E, Salmerón J. The effect of vitamin D supplementation on serum lipids in postmenopausal women with diabetes: A randomized controlled trial. Clin Nutr 2015;34(5):799-804

11. Bayani MA, Akbari R, Banasaz B, Saeedi F. Status of vitamin-D in diabetic patients. Caspian J Intern Med 2014;5:40-2.

12. Goswami R, Gupta N, Ray D, Singh N, Tomar N. Pattern of 25-hydroxy vitamin $\mathrm{D}$ response at short ( 2 month) and long (1 year) interval after 8 weeks of oral supplementation with cholecalciferol in Asian Indians with chronic hypovitaminosis D. Br J Nutr 2008;100(6):526-9.

13. Palomer X, González-Clemente JM, Blanco-Vaca F, Mauricio D. Role of vitamin D in the pathogenesis of Type 2 diabetes mellitus. Diabetes Obes Metab 2008;10(3):185-97.

14. Boucher BJ, Mannan N, Noonan K, Hales CN, Evans SJ. Glucose intolerance and impairment of insulin secretion in relation to vitamin $\mathrm{D}$ deficiency in east London Asians. Diabetologia 1995;38(1):1239-45.

15. Isaia G, Giorgino R, Adami S. High prevalence of hypovitaminosis D in female Type 2 diabetic population. Diabetes Care 2001;24(8):1496.

16. Mattila C, Knekt P, Männistö S, Rissanen H, Laaksonen MA, Montonen J, et al. Serum 25-hydroxyvitamin D concentration and subsequent risk of Type 2 diabetes. Diabetes Care 2007;30(10):2569-70.

17. Mitri J, Dawson-Hughes B, Hu FB, Pittas AG. Effects of vitamin D and calcium supplementation on pancreatic $\beta$ cell function, insulin sensitivity, and glycemia in adults at high risk of diabetes: The calcium and vitamin D for diabetes mellitus (CaDDM) randomized controlled trial. Am J Clin Nutr 2011;94(2):486-94.

18. Song Y, Wang L, Pittas AG, Del Gobbo LC, Zhang C, Manson JE, et al. Blood 25-hydroxy vitamin D levels and incident Type 2 diabetes: A meta-analysis of prospective studies. Diabetes Care 2013;36(5):1422-8.

19. Chakhtoura M, Azar ST. The role of vitamin D deficiency in the incidence, progression, and complications of Type 1 diabetes mellitus. Int J Endocrinol 2013;2013:148673.

20. Pittas AG. The role of vitamin D and calcium in Type 2 diabetes. A systematic review and meta-analysis. J Clin Endocrinol Metab 2007;92(6):2017-29.

21. Bajaj S, Singh RP, Dwivedi NC, Singh K, Gupta A, Mathur M.
Vitamin D levels and microvascular complications in Type 2 diabetes. Indian J Endocrinol Metab 2014;18(4):537-41.

22. Chaudhary S, Thukral A, Tiwari S, Pratyush DD, Singh SK. Vitamin D status of patients with Type 2 diabetes and sputum positive pulmonary tuberculosis. Indian J Endocrinol Metab 2013;17 Suppl 3:S670-3.

23. Pittas AG, Dawson-Hughes B, Li T, Van Dam RM, Willett WC, Manson JE, et al. Vitamin D and calcium intake in relation to Type 2 diabetes in women. Diabetes Care 2006;29(3):650-6.

24. Mathieu C, Gysemans C, Giulietti A, Bouillon R. Vitamin D and diabetes. Diabetologia 2005;48(7):1247-57.

25. Kalsi A, Singh S, Taneja N, Kukal S, Mani S. Current treatments for Type 2 diabetes, their side effects and possible complementary treatments. Int J Pharm Pharm Sci 2014;7:13-8.

26. Karhapää P, Pihlajamäki J, Pörsti I, Kastarinen M, Mustonen J, Niemelä $\mathrm{O}$, et al. Diverse associations of 25-hydroxyvitamin D and 1,25-dihydroxy-vitamin D with dyslipidaemias. J Intern Med 2010;268(6):604-10.

27. Jorde R, Grimnes G. Vitamin D and metabolic health with special reference to the effect of vitamin D on serum lipids. Prog Lipid Res 2011;50(4):303-12

28. Bi X, Tey SL, Leong C, Quek R, Henry CJ. Prevalence of vitamin D deficiency in Singapore: Its implications to cardiovascular risk factors. PLoS One 2016;11(1):e0147616.

29. Dobiásová M, Frohlich J. The plasma parameter log (TG/HDL-C) as an atherogenic index: Correlation with lipoprotein particle size and esterification rate in apoB-lipoprotein-depleted plasma (FER(HDL)). Clin Biochem 2001;34(7):583-8.

30. Saedisomeolia A, Taheri E, Djalali M, Moghadam AM, Qorbani M. Association between serum level of vitamin D and lipid profiles in Type 2 diabetic patients in Iran. J Diabetes Metab Disord

31. S(entkalt3 (1h) G..Jayprakash A, Swathi KS. The effect of sun light exposure on prediabetic patients in South Indian population. Int J Pharm Pharm Sci 2014;6(11):107-110.

32. Wang JH, Keisala T, Solakivi T, Minasyan A, Kalueff AV, Tuohimaa P. Serum cholesterol and expression of ApoAI, LXRbeta and SREBP2 in vitamin D receptor knock-out mice. J Steroid Biochem Mol Biol 2009;113(3-5):222-6.

33. Querfeld U, Hoffmann MM, Klaus G, Eifinger F, Ackerschott M, Michalk D, et al. Antagonistic effects of vitamin D and parathyroid hormone on lipoprotein lipase in cultured adipocytes. J Am Soc Nephrol 1999;10(10):2158-64. 